\title{
Optimal taxonomic groups for biodiversity assessment: A meta-analytic approach
}

\author{
Martin J. Westgate ${ }^{1,2}$, Ayesha I. T. Tulloch ${ }^{1}$, Philip S. Barton ${ }^{1}$, Jennifer C. Pierson \& \\ David B. Lindenmayer ${ }^{1}$ \\ ${ }^{1}$ The Fenner School of Environment and Society, The Australian National University, \\ Canberra ACT 0200, Australia \\ ${ }^{2}$ Corresponding author: martin.westgate@anu.edu.au
}

Keywords: species richness, species composition, surrogates and indicators, spatial scale

This is the author's version of a manuscript accepted for publication by Ecography on $28^{\text {th }}$ February 2016.

\begin{abstract}
A fundamental decision in biodiversity assessment is the selection of one or more study taxa, a choice that is often made using qualitative criteria such as historical precedent, ease of detection, or available technical or taxonomic expertise. A more robust approach would involve selecting taxa based on the a priori expectation that they will provide the best possible information on unmeasured groups, but data to inform such hypotheses are often lacking. Using a global meta-analysis, we quantified the proportion of variability that each of 12 taxonomic groups (at the Order level or above) explained in the richness or composition of other taxa. We then applied optimization to matrices of pairwise congruency to identify the best set of complementary surrogate groups. We found that no single taxon was an optimal surrogate for both the richness and composition of unmeasured taxa if we used simple methods to aggregate congruence data between studies. In contrast, statistical methods that accounted for well-known drivers of cross-taxon congruence (spatial extent, grain size, and latitude) lead to the prioritization of similar surrogates for both species richness and composition. Advanced statistical methods were also more effective at describing known ecological relationships between taxa than simple methods, and show that congruence is typically highest between taxonomically and functionally dissimilar taxa. Birds and vascular plants were most frequently selected by our algorithm as surrogates for other taxonomic groups, but the extent to which any one taxon was the 'optimal' choice of surrogate for other biodiversity was highly contextdependent. In the absence of other information - such as in data-poor areas of the globe, and under limited budgets for monitoring or assessment - ecologists can use our results to assess which taxa are most likely to reflect the distribution of the richness or composition of 'total' biodiversity.
\end{abstract}




\section{INTRODUCTION}

Attempts to halt biodiversity loss have received considerable scientific and political attention, and conservation policy addressing this issue has become correspondingly prevalent (Lung et al. 2014, Sachs et al. 2009). However, efforts to conserve biodiversity require that we can efficiently determine where species are located (Balmford and Gaston 1999), an expectation that is known to be flawed in several important ways. In particular, ecological research is heavily biased towards Europe and North America, with the biodiversity of other regions being comparatively poorly described (Martin et al. 2012). Further, different components of biodiversity (i.e. alpha versus beta diversity) are differentially affected by scale or ecological gradients (Barton et al. 2013, Dumbrell et al. 2008, Supp and Ernest 2014), confounding attempts to predict the location of biodiversity hotspots. Finally, cost and logistical constraints to monitoring and surveying budgets mean that assessment of assemblage structure is typically only possible for a subset of taxonomic groups (although see Barlow et al. 2007, Gardner et al. 2008, Kessler et al. 2011, Landeiro et al. 2012). Therefore, a key problem for evaluating biodiversity policy is: How do we quantify 'total' biodiversity in a robust but cost-effective manner?

Biodiversity surrogates provide a tractable and frequently-used alternative to comprehensive monitoring or assessment of multiple taxa (Sarkar and Margules 2002). In a broad sense, ecological surrogacy involves the assessment of one or more components of an environment or its biota, with the assumption that variation in the surrogate reflects change in an important but difficult-to-measure attribute (Hunter Jr et al. 2016). In this paper, we focus on a class of surrogates that use measurements of one or more taxonomic groups to infer the properties of unmeasured taxa, and thus a broader sample of biodiversity (Araujo et al. 2004, McGeogh 1998). The potential benefit to conservation science of identifying taxa that can be used as consistent biodiversity surrogates is enormous, as alternative approaches that utilize abiotic surrogates (such as climate or topography; Faith 2003, McArthur et al. 2010) are liable to error where biological and environmental diversity respond to different processes or at different rates (Lawler et al. 2015). However, biodiversity surrogates are required to display strong spatial covariation with other taxa (i.e. high 'cross-taxon congruence'; Mellin et al. 2011), which is a challenging criterion as congruence can vary between locations (Heino 2014), over time (Tulloch et al. 2016), and with changes in spatial scale (Hess et al. 2006, Westgate et al. 2014). Further, the best methods we have for evaluating biodiversity surrogates use site-based complementarity (Rodrigues and Brooks 2007), but this method is highly sensitive to differences in analytical assumptions (Grantham et al. 2010), hindering effective synthesis. Consequently, there is no consensus as to which taxa are useful biodiversity surrogates across a broad range of circumstances; how many taxonomic groups might be needed to ensure that 'total' biodiversity is adequately represented in a given survey; or whether these associations are robust across distinct indices of assemblage 
structure (i.e. richness vs. composition). Answering these questions would be a critical first step towards identifying robust surrogates for biodiversity monitoring and assessment.

In this paper, we use a meta-analytic approach (Arnqvist and Wooster 1995) to identify optimal biodiversity surrogates, by finding the best set of complementary surrogates for the richness or composition of other taxonomic groups. Previous work has shown that monitoring and management programs that ignore redundancy between species or actions will be more costly and less efficient (Chadès et al. 2015, Tulloch et al. 2013). This is because some monitoring or management actions provide benefits for the same groups (e.g. due to similarities between groups in threats, resource use and environmental niche overlap), and so choosing to monitor or survey a second taxon that provides similar information to the first will provide little additional benefit (Moilanen 2008). Complementarity-based methods also provide greater insight and predictive power than studies that attempt to predict the distribution of 'total' biodiversity by grouping unlike taxa (Rodrigues and Brooks 2007). Our approach attempts to generalize earlier work that quantified the surrogacy value of individual taxonomic groups such as birds (Eglington et al. 2012) or plants (Castagneyrol and Jactel 2012), by quantifying all pairwise associations between many different plant and animal taxa (at the Order level or above) in a single analysis. Specifically, we address two questions:

1. To what extent does pairwise cross-taxon congruence vary between studies in different locations or at different spatial scales?

2. Is any single taxon an effective surrogate for both the richness and composition of unmeasured groups?

Describing networks of spatial associations between taxa is an important goal for guiding both research and biodiversity management. Such information can influence our understanding of the distribution and drivers of biodiversity (Wisz et al. 2013), suggest strong ecological connections deserving of further study (Hawkins and Porter 2003), and identify potential surrogates for the diversity of unobserved taxa (Rodrigues and Brooks 2007). Conversely, failing to investigate the assumption that one taxon can adequately represent 'total' biodiversity avoids the effort of identifying and testing alternative surrogates (Lindenmayer et al. 2015), but magnifies our uncertainty in how biodiversity is distributed and how it should be managed (Field et al. 2004). Here, we synthesize patterns of pairwise cross-taxon congruence for the first time. Our results generate new insights as to the potential strengths and limitations of cross-study comparison for identifying optimal biodiversity surrogates. 


\section{METHODS}

\section{Article identification}

We used the ISI web of knowledge to search for articles that contained: (1) the word 'species' and (2) any of the words 'congruence', 'surrogate' or 'indicator', and (3) any of the words 'assemblage', 'richness' or 'composition'. Further, entries had to be articles or reviews of terrestrial or freshwater ecosystems in the topic 'Environmental sciences and ecology' that were written in English. We supplemented this list of 4698 articles (accessed on $20^{\text {th }}$ February 2015) with data from a further two sources. First, six of the articles identified by our search were themselves reviews or meta-analyses that contained potentially relevant data (Castagneyrol and Jactel 2012, Eglington et al. 2012, Heino 2010, Lewandowski et al. 2010, Velghe and Gregory-Eaves 2013, Wolters et al. 2006). We therefore manually searched all articles cited by these reviews to identify any further information that we might have missed in our own search. Second, we added all relevant data from an earlier meta-analysis on a related topic (Westgate et al. 2014). We investigated any articles from these sources that appeared to contain relevant data on the basis of their title or abstract. Our criteria were to exclude any data that used only the 'higher taxon' method of biodiversity categorization and surrogacy (sensu Gaston and Williams 1993), that compared animal diversity only to plant structural attributes (e.g. Barton et al. 2014), or that focused on the response of biodiversity to management or ecological processes (unless they compared the performance of multiple taxa, e.g. Barlow et al. 2007).

\section{Extraction of congruence data}

We collected data on cross-taxon congruence for two distinct metrics of assemblage structure: species richness and species composition (Fig. 1a). Congruence in species richness is the degree to which the number of species from taxon A co-varies with the number of species from taxon $B$, when assessed at a set of survey locations (sites). Conversely, congruence in species composition tests the correlation between two distance matrices (one per taxon), each of which describes the (dis)similarity in species occurrences or abundances between pairs of sites for a given taxon.

Congruence in species richness can be assessed using Pearson's correlations or linear regression, while congruence in composition is typically assessed using Mantel tests. 
Figure 1: Flow diagram illustrating the key stages of our analytical approach, including a) the types of data extracted from empirical articles including a description of their derivation; $b$ ) examples of how data from groups of species with different degrees of taxonomic validity and frequency of occurrence were selected and collated, and c) an overview of the modelling and optimization approaches used to derive our conclusions. See text for a full description of each stage.
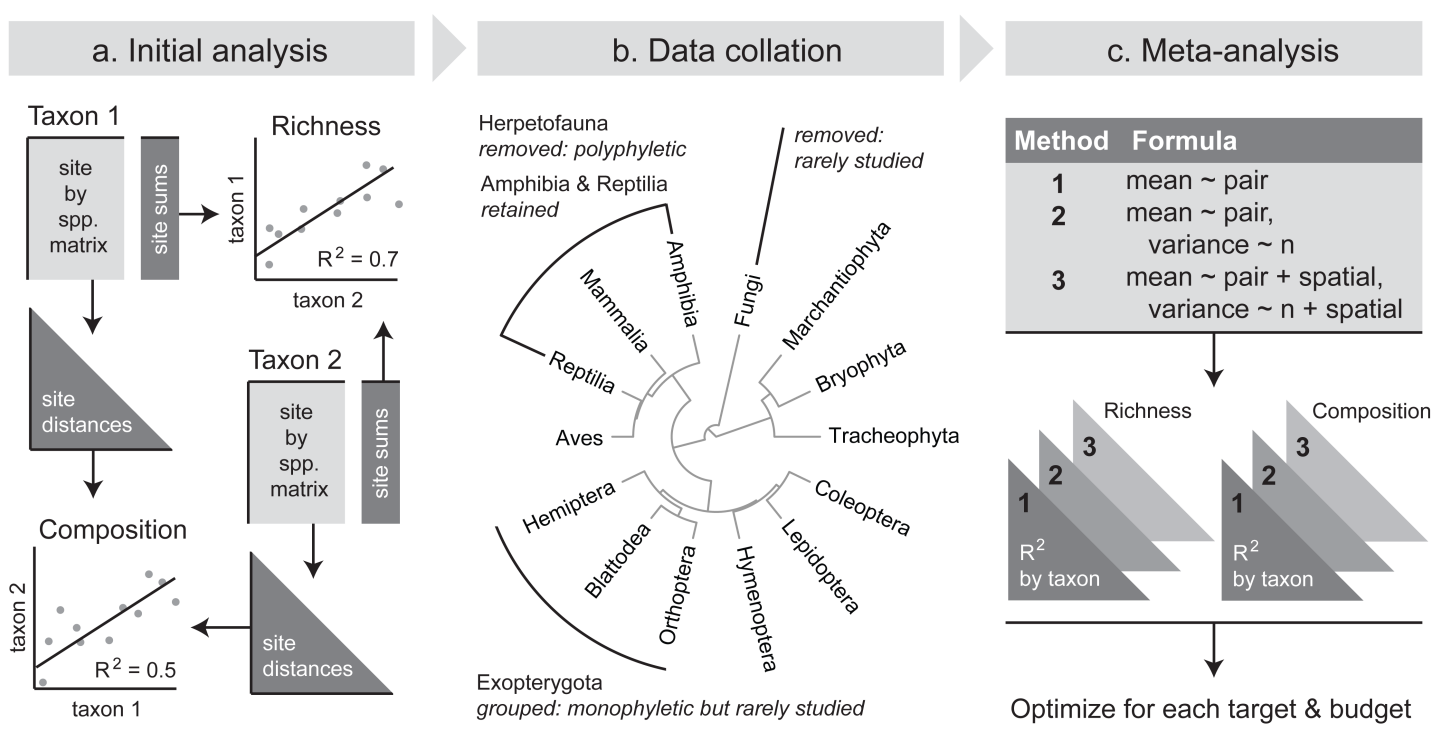

We used the $\mathrm{R}^{2}$ statistic to quantify the degree of congruence (i.e. to summarize the findings of the tests described above) between each pair of taxa. That is, all of our analyses investigated the proportion of variance explained by comparing the richness or composition of one taxon against the same metric for a second taxon. Although the correlation coefficient (Pearson's r) was a more commonly reported statistic in the articles that we studied, we used $\mathrm{R}^{2}$ because we were more interested in the strength of the association between metrics than their sign. The distribution of observed $\mathrm{R}^{2}$ values in our dataset was strongly skewed towards zero, and so we logit-transformed this variable after adjusting values that were exactly zero to an arbitrary but very low value using the formula:

$$
\mathrm{y}=10^{-(a+1)}
$$

Where $a$ is the number of decimal places to which the initial statistic was originally reported. Our process for adjusting values that were exactly one was the same, but using one minus the result of the above formula.

While gathering data on our variables of interest (i.e. congruence in richness or composition), we also collected four 'predictor' variables that quantified attributes of the design of each study. First, we recorded the sample size of the analysis used to calculate each statistic. We extracted this variable separately for each observation (i.e. each $\mathrm{R}^{2}$ value) in our dataset, as estimates of congruence for distinct pairs of taxa within the same study were often calculated from different subsets of data. Second, 
we recorded the grain size of each study as the size of the smallest survey unit subject to investigation. Where the size of the study unit was given as a transect length (rather than as an area), we assumed each transect to have a width of ten metres. Finally, we identified the coordinates of the corners of the study region(s) from each paper, and used this information to calculate two metrics. We took the total area of terrestrial surface within the study region (measured in square kilometers) as the spatial extent of that study, while the centroid of the bounding box was used to give the latitude of that region. We chose these variables because they have each been shown to influence patterns in cross-taxon congruence in studies similar to our own (Ekroos et al. 2013, Hess et al. 2006, Westgate et al. 2014).

\section{Data collation}

Our goal was to assess the degree of congruence between pairs of taxa, but two factors constrained our ability to compare results from different studies (see Fig. 1b for a diagrammatic representation). First, not all groups of species were taxonomically valid. For example, the term 'Herpetofauna' is a common ecological classification for amphibians and reptiles, but this group is polyphyletic. We dealt with this problem by excluding all groups of species that did not refer to taxonomically distinguishable entities. Second, not all taxa are equally well studied, leaving gaps in the dataset. Because pairwise correlations (i.e. our response variables) are largely intransitive (Langford et al. 2001), we were unable to infer the degree of association between pairs of taxa where empirical data were absent. Where possible, we avoided this problem by grouping taxa into higher monophyletic clades for which sufficient data were available (e.g. Exopterygota in Fig. 1b). However, grouping of sister taxa could lead to some taxa being compared against themselves. For example, Schall and Pianka (1978) provide data on the association between richness of snakes and lizards, taxa that were both classified as Reptilia in our analysis. In the absence of a method for incorporating data on the amount of information that each taxon provided on itself, we removed any such sets from our analysis. Following collation, any taxa that lacked data on their association with all other taxa in the dataset were removed (e.g. Fungi).

\section{Question 1: Influence of study design on pairwise congruence}

Our meta-analysis stage (Fig. 1c) was designed to summarize the many studies on each pair of taxa into a single value - termed $c_{i j}(k)$ - which would give the estimated amount of richness or composition information that one group $i$ provides on another group $j$ if assessed, using method $k$ for analysis. Because there is no single 'best' way to aggregating congruence data between studies, however, we investigated three methods of increasing sophistication to estimate $c_{i j}$. Each method returned a symmetric matrix with $m$ rows and columns, where each cell contained estimated values of $c_{i j}(k)$ and $m$ was the number of taxonomic groups included in the analysis. We calculated each of our three methods on two distinct subsets of data; i.e. for both species richness $(n=1189$ correlations from 134 locations in 116 articles) and separately for congruence in species composition $(n=480$ correlations from 36 
locations in 31 articles). This was necessary because $\mathrm{R}^{2}$ values cannot be directly compared between Mantel tests (which are commonly used to assess congruence in species composition) and correlation or regression-based analyses (used to asses congruence in species richness; Legendre et al. 2015). We ran all of our analysis in the R statistical environment (R Core Development Team 2015).

Each of our three methods used a different approach to calculate the estimated $\mathrm{R}^{2}$ value between each pair of taxa. Method 1 was simply the mean $\mathrm{R}^{2}$ between each pair. Method 2 was the expected $\mathrm{R}^{2}$ from a Gaussian (normal) GAMLSS model (Generalized Additive Model for Location, Scale and Shape; Rigby and Stasinopoulos 2005), in which pair-specific means were calculated using random effects, while variance in $\mathrm{R}^{2}$ was modeled as a function of sample size using a spline with one degree of freedom. That is, our estimates of pairwise congruence from this model accounted for the fact that variance in $\mathrm{R}^{2}$ would be high when the sample size of the study used to calculate each statistic was low. Method 3 was the expected $\mathrm{R}^{2}$ from another GAMLSS model, in which spatial extent (in $\mathrm{km}^{2}$ ), distance from the equator (degrees latitude), their interaction, and grain size $\left(\mathrm{km}^{2}\right)$ were allowed to influence both the mean and variance in congruence estimates, while sample size was also included as a predictor of variance. This was to explicitly account for the expectation from earlier work that each of these variables can influence cross-taxon congruence (Hess et al. 2006).

To assess the extent to which estimates of cross-taxon congruence varied depending on our method of data aggregation, we calculated Mantel tests between each of the three species-by-species matrices returned by the above methods. High correlations between matrices would imply low influence of study-related covariates on crosstaxon congruence.

\section{Question 2: Optimal biodiversity surrogates}

We used the pairwise congruence matrices that we derived from our three data aggregation methods (described above) in a series of optimizations to find the set of complementary taxonomic groups that maximized total representation of target taxa for each matrix (Chadès et al. 2015, Tulloch et al. 2016, Tulloch et al. 2013). Because some taxa will be useful surrogates for some objectives but not others, we explored three groups of target taxa: all taxa, only vertebrates, or only invertebrates.

Using the optimization approach of Tulloch et al. (2016), we defined a set benefit function $B(k, Z)$ that maximizes the complementary biodiversity assessment benefits $c$ of a set of $Z$ taxonomic groups for a set of target groups $T$ (i.e. either all 11 groups, or just 4 vertebrate taxa, or just 6 invertebrate taxa), and a given congruence aggregation method $k$ (leading to 18 possible options in this study: 3 methods applied to calculating congruence in 2 metrics for 3 targets). We then found the taxonomic group $i$ that maximized the proportional variance explained in target taxonomic group $j$, before summing the maximal congruence values, $c_{i j}$, across all target taxa $T$ : 


$$
B(k, Z)=\sum_{j \in T} \max _{i \in Z} c_{i j}(k) \text { with } Z \subseteq S,
$$

where $c_{i j}(k)$ is an estimated $\mathrm{R}^{2}$ value between 0 and 1 (inclusive), and $Z$ is a subset of the set of all possible taxonomic groups $S$. Each taxon was assumed to perfectly represent itself; ergo the diagonal of each congruence matrix was set to one. We also set the constraint that only one taxon $i$ can contribute to target species $j$. This means that if a taxon $i$ is selected, the target taxa $j$ for which it maximizes $c_{i j}(k)$ contributes benefits to $B(k, Z)$ once only. This has the consequence that increasing the summed benefits $B(k, Z)$ of a selected group of surrogates $Z$ requires the addition of a new taxon $i$, representing the maximal congruency values for different taxa $j$. In doing so, the equation includes complementarity in cross-taxon congruence to ensure that redundant taxonomic groups (i.e. those with no additional benefits of monitoring due to representing taxa that are already well-represented by another group) are not selected in the best sets of taxonomic groups.

The best complementary set of taxonomic groups $Z^{*}$ is the set that maximizes $B(k, Z)$, the sum of the highest congruencies $c_{i j}$ for each taxonomic group, within a given budget constraint, formally:

$Z^{*}(k)=\operatorname{argmax}_{Z \subseteq s}\{B(k, Z)$ such that $\operatorname{tax} a(Z) \leq$ budget $\}$

where $\operatorname{tax} a(Z)$ is the number of taxonomic groups selected in set $Z$ for monitoring, and argmax finds the set of taxa $Z^{*}$ for which function $B(k, Z)$ attains the maximum value. We defined the budget as the desired number of taxonomic groups to be selected for assessment. We tested all integer budgets from one (a single best taxonomic group selected) to six groups, and identified optimal taxa via an exhaustive search of all possible taxon combinations.

To assess whether we might choose the same taxa for representing species richness as we would to represent species composition (when assessed against the same target group), we tested the overlap between pairs of optimization results using Fisher's exact tests. This approach tested whether richness and composition datasets were significantly more likely to select the same taxa than we would expect by chance (i.e. that the odds ratio was significantly $>1$ ). We also tested the improvement in monitoring power that each of the selected surrogate sets (for different budgets, and using different congruency methods) gained compared with a randomly selected set of surrogates (see Supporting Material). 


\section{RESULTS}

\section{Question 1: Influence of study design on pairwise congruence}

Patterns of mean cross-taxon congruence differed markedly depending on whether the metric under investigation was species richness or species composition. For species richness, mean values showed high congruence of flies (Diptera) with three other taxa (Hymenoptera, Exopterygota \& Tracheophyta), while the strongest associations between vertebrate taxa were between Amphibia and either Aves or Mammalia (Fig. $2 \mathrm{a})$. Mean congruence in species composition $\left(0.08, \max \mathrm{R}^{2}=0.49\right)$ was lower than for species richness $\left(0.12, \max \mathrm{R}^{2}=0.37\right)$, a result that is not atypical when comparing Mantel and Pearson's correlations. The single strongest association in species composition was between Exopterygota and Plantae, while both Coleoptera and Aves displayed a large number of strong associations with other taxa (Fig 2d).

Several predictor variables were significantly associated with changes in cross-taxon congruence (Table 1). Testing the effect of sample size on variance in $\mathrm{R}^{2}$ values using GAMLSS (method 2) showed significantly higher variance in studies with small sample size for species richness data, but not species composition. Adding spatial effects to the model (extent, grain size and location; method 3) revealed strong effects of these covariates on both the mean and variance of cross-taxon congruence. For species richness, $\mathrm{R}^{2}$ values were significantly higher at large spatial extents and high latitudes. There also was a weak effect suggesting increasing $\mathrm{R}^{2}$ at larger grain sizes, but this effect was significantly lower at high latitudes. Spatial extent was the only variable to influence the variance in $\mathrm{R}^{2}$ values for species richness. For species composition, $\mathrm{R}^{2}$ was higher at large spatial extents, and higher latitudes, but the effect of latitude was lower for larger extents. Variance in $\mathrm{R}^{2}$ for species composition was significantly lower at larger extents, at high latitudes, and for small sample sizes, but the reduction in variance with increasing extent was moderated at high latitudes. Finally, grain size had no effect on the mean or the variance in $\mathrm{R}^{2}$ for species composition. 
Figure 2: Cross-taxon congruence in species richness (a-c) and composition (d-f), estimated using three methods of increasing complexity.
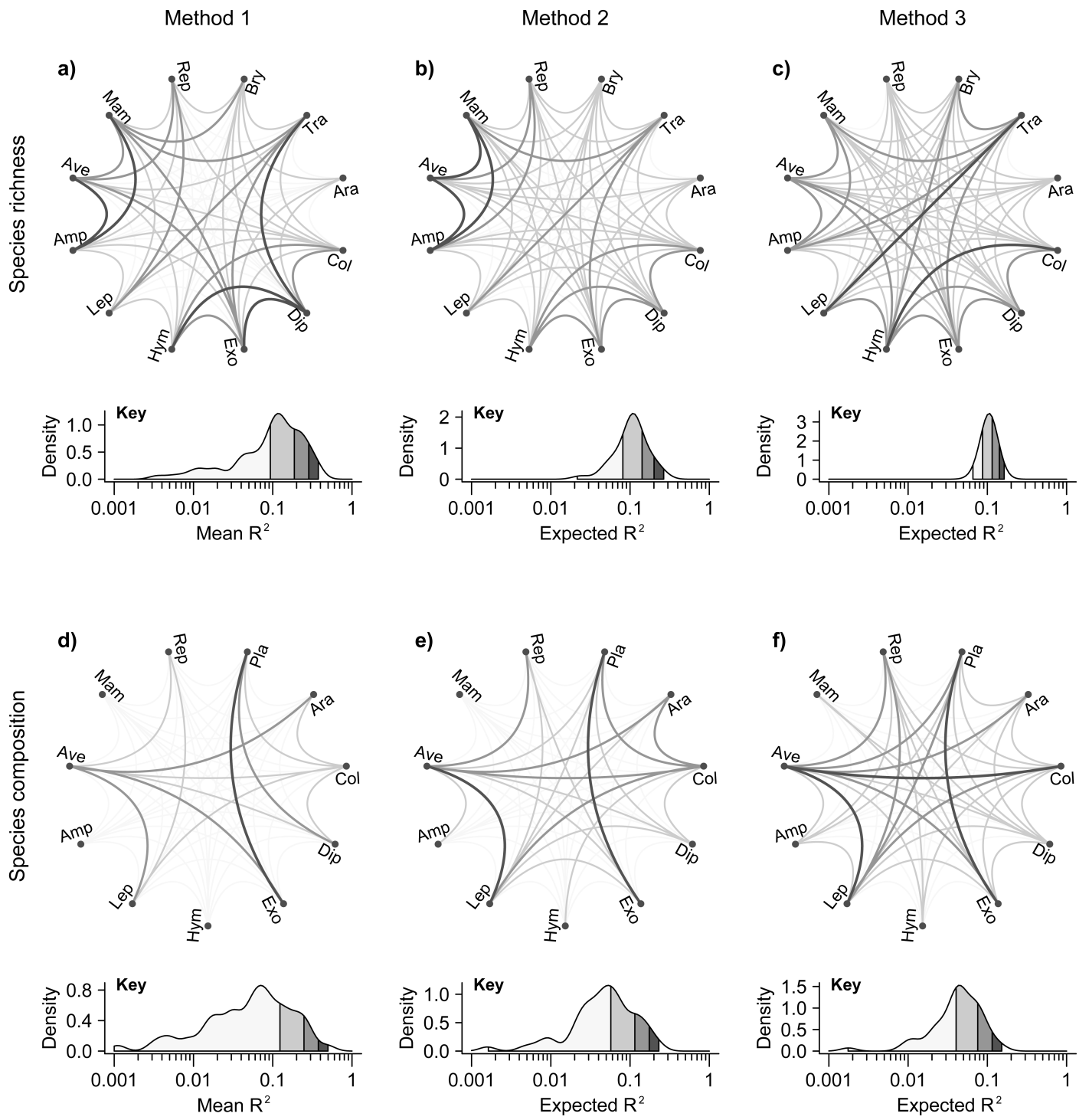

Methods for deriving $R^{2}$ values are as follows: 1) mean value from all studies on each pair of taxa; 2) estimated value after accounting for effects of sample size on variance in observed $R^{2}$ values using a GAMLSS model; 3) estimated value after accounting for effects of spatial covariates and sample size on mean and variance using a GAMLSS model (see text for full details). Line breaks divide the data into four equally sized regions, shown on the log scale in each key. Key to taxonomic labels (clockwise from top right): Pla = Plantae, Bry = Bryophyta, Tra $=$ Tracheophyta, Ara = Arachnida, Col=Coleoptera, Dip = Diptera, Exo= Exopterygota, Hym = Hymenoptera, Lep = Lepidoptera, Amp = Amphibia, Ave = Aves, Mam $=$ Mammalia, Rep $=$ Reptilia . 
Table 1: Effects of covariates on the mean and variance in $R 2$ values.

\begin{tabular}{|c|c|c|c|c|}
\hline Model & Response & Term & Richness & Composition \\
\hline \multicolumn{5}{|c|}{ Method 2} \\
\hline & Mean & Intercept & $-2.15(\mathrm{SE}=0.08, \mathrm{P}<0.001)$ & $-3.05(\mathrm{SE}=0.10, \mathrm{P}<0.001)$ \\
\hline & Variance & Intercept & $2.44(\mathrm{SE}=0.05, \mathrm{P}<0.001)$ & $1.94(\mathrm{SE}=0.07, \mathrm{P}<0.001)$ \\
\hline & & Sample size & $-0.23(\mathrm{SE}=0.06, \mathrm{P}<0.001)$ & $-0.08(\mathrm{SE}=0.07, \mathrm{P}=0.25)$ \\
\hline \multicolumn{5}{|c|}{ Method 3} \\
\hline & Mean & Intercept & $-2.08(\mathrm{SE}=0.07, \mathrm{P}<0.001)$ & $-3.26(\mathrm{SE}=0.10, \mathrm{P}<0.001)$ \\
\hline & & Extent & $0.49(\mathrm{SE}=0.15, \mathrm{P}=0.001)$ & $0.29(\mathrm{SE}=0.11, \mathrm{P}=0.007)$ \\
\hline & & Latitude & $0.30(\mathrm{SE}=0.07, \mathrm{P}<0.001)$ & $0.74(\mathrm{SE}=0.07, \mathrm{P}<0.001)$ \\
\hline & & Grain & $0.27(\mathrm{SE}=0.14, \mathrm{P}=0.06)$ & $0.10(\mathrm{SE}=0.08, \mathrm{P}=0.23)$ \\
\hline & & Grain:Latitude & $-0.33(\mathrm{SE}=0.07, \mathrm{P}<0.001)$ & $-0.15(\mathrm{SE}=0.06, \mathrm{P}=0.01)$ \\
\hline & Variance & Intercept & $2.19(\mathrm{SE}=0.05, \mathrm{P}<0.001)$ & $1.86(\mathrm{SE}=0.07, \mathrm{P}<0.001)$ \\
\hline & & Extent & $-0.45(\mathrm{SE}=0.12, \mathrm{P}<0.001)$ & $-0.30(\mathrm{SE}=0.08, \mathrm{P}<0.001)$ \\
\hline & & Latitude & $-0.01(\mathrm{SE}=0.05, \mathrm{P}=0.92)$ & $-0.18(\mathrm{SE}=0.05, \mathrm{P}=0.001)$ \\
\hline & & Grain & $0.11(\mathrm{SE}=0.11, \mathrm{P}=0.36)$ & $-0.07(\mathrm{SE}=0.07, \mathrm{P}=0.31)$ \\
\hline & & Sample size & $-0.08(\mathrm{SE}=0.06, \mathrm{P}=0.17)$ & $-0.33(\mathrm{SE}=0.08, \mathrm{P}<0.001)$ \\
\hline & & Grain:Latitude & $0.05(\mathrm{SE}=0.06, \mathrm{P}=0.35)$ & $0.26(\mathrm{SE}=0.05, \mathrm{P}<0.001)$ \\
\hline
\end{tabular}

Table shows mean values of smoothed coefficients. Colons designate an interaction between two terms.

Our three methods for amalgamating $\mathrm{R}^{2}$ values between studies showed marked differences in patterns of congruence for species richness, but not species composition. For species richness, highest congruence values using method one (mean congruence; Fig. 2a) occurred between three vertebrate taxa (Amphibia, Aves \& Mammalia), as well as between Diptera and three other groups (Tracheophyta, Exopterygota and Hymenoptera). Method two (GAMLSS model to account for sample size effect) gave similar results to method one (Mantel $=0.91$; Table 2 ), but showed that the strong influence of Diptera observed using mean values (method one) was probably an artifact of small sample size (Fig. 2b). Method three (GAMLSS model to account for spatial effects) was less correlated with either method one (0.62) or method $2(0.70)$ than those methods were with each other $(0.90)$. Correspondingly, method three showed different patterns of pairwise congruence, with highest congruence occurring between two pairs that were not selected at all by earlier methods (Tracheophyta and Lepidoptera, Coleoptera and Hymenoptera; Fig. 2c). For species composition, the change in highly-congruent taxa with increasingly complex methods was not so marked as for species richness (the lowest correlation between methods for this metric was 0.84; Table 2). Instead, five of the 11 taxonomic groups (Plantae, Coleoptera, Exopterygota, Lepidoptera and Aves) showed stronger-thanaverage connections to one or more taxa under all three methods (Fig. 2d-f). The 
amount of data available for each pair of taxa was not a significant predictor of crosstaxon congruence, for any metric or method (Table 2).

\section{Question 2: Identifying optimal surrogates}

The optimal taxon for maximizing representation of all taxa was strongly dependent on the target and on the metric used to assess cross-taxon congruence (richness or composition; Fig. 3). Where the goal was to optimally represent vertebrates, methods one and two (mean $\mathrm{R}^{2}$ and predicted GAMLSS $\mathrm{R}^{2}$ adjusting for low sample size, respectively) showed that Aves and Mammalia were optimal taxa to represent the composition of unobserved vertebrate taxa, while Amphibia and Reptilia were the best surrogates for the richness of other vertebrates. In contrast, method three (GAMLSS model including sample size and spatial covariates) showed that Aves was the optimal taxon to represent both the richness and composition of other vertebrates. For our remaining targets (optimal representation of invertebrates, or all taxa), analysis of species composition gave consistent results, selecting Coleoptera and Exopterygota as the best two surrogate taxa for invertebrates under all three methods, and Aves and Plantae as the best surrogates for all taxa. However, no two methods selected the same pair of optimal taxa for species richness, even when assessed against the same target. Sensitivity analyses revealed that the optimal surrogate set always had higher monitoring power compared with a randomly selected surrogate set, and that this difference was greater when there were specific targets such as only vertebrates or only invertebrates, compared with the goal of assessing 'total' biodiversity (Supporting Material).

Analysis of the degree of overlap between the best set of surrogate taxa for our richness versus composition datasets (using Fishers' exact tests on odds ratios) showed marked differences between our richness and composition results. In fact, when using mean $\mathrm{R}^{2}$ values (method 1 ) to select optimal surrogates among all species, optimal solutions for species richness were negatively associated with species composition solutions (Fig. $2 \mathrm{~g}$, odds ratio $=0.18$ ), suggesting that the same taxa could not be optimal surrogates for both metrics. Nor was there significant overlap among selected taxa for any target (all species, vertebrates or invertebrates) using either of our first two methods. However, after accounting for study-related spatial variation (method three), there was a significant relationship among the richness and composition datasets when assessing overlap in the identity of optimal taxa to represent all other species (odds ratio $=8.4, \mathrm{P}<0.001$; Fig 3i). There also was a strong association between methods when selecting optimal surrogates for vertebrate taxa (odds ratio $=3.53$; Fig. $3 \mathrm{c}$ ), but this was not statistically significant $(\mathrm{P}=0.28)$, possibly due to small sample size $(n=12)$. 
Figure 3: Taxa selected under a range of targets, budgets, and methods of accounting for study-related bias.
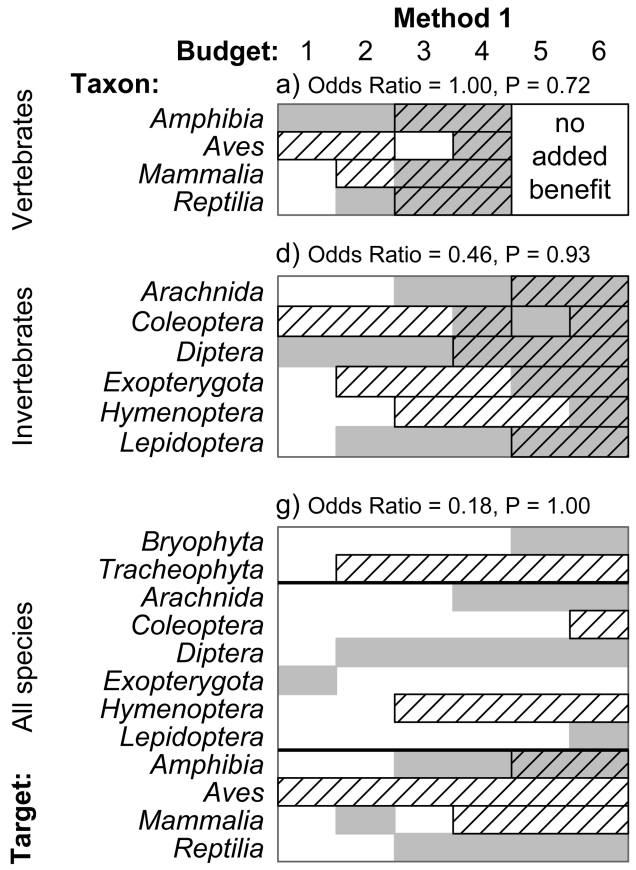
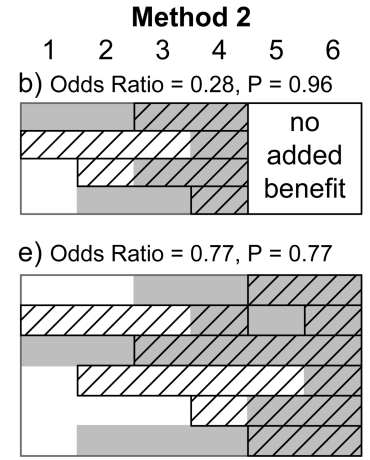

h) Odds Ratio $=0.96, P=0.63$

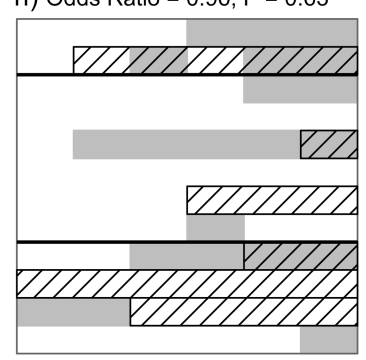

Method 3

$\begin{array}{llllll}1 & 2 & 3 & 4 & 5 & 6\end{array}$

c) Odds Ratio $=3.53, \mathrm{P}=0.28$

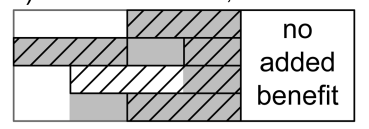

f) Odds Ratio $=0.77, \mathrm{P}=0.77$

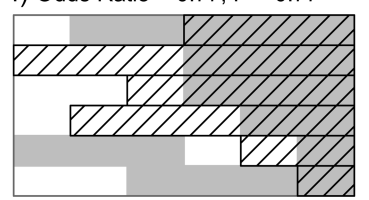

i) Odds Ratio $=8.40, \mathrm{P}<0.001$

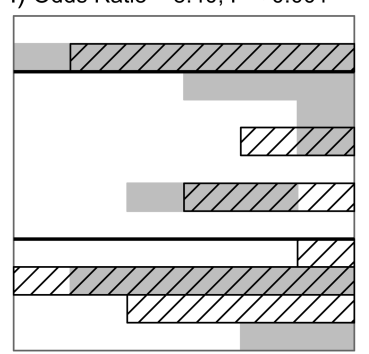

Grey fill represents taxa selected for representativeness in the species richness dataset, while dashed lines represent taxa selected in the species composition dataset. For species composition results, Tracheophyta incorporates data for all vascular and non-vascular plants.

Table 2: Mantel correlations between different methods for estimating pairwise cross-taxon congruence

\begin{tabular}{lcccc} 
& Count & Method 1 & Method 2 & Method 3 \\
\cline { 2 - 5 } Count & & $-0.08(\mathrm{P}=0.62)$ & $0.05(\mathrm{P}=0.38)$ & $0.10(\mathrm{P}=0.31)$ \\
Method 1 & $0.14(\mathrm{P}=0.14)$ & & $0.92(\mathrm{P}<0.001)$ & $0.84(\mathrm{P}<0.001)$ \\
Method 2 & $0.17(\mathrm{P}=0.10)$ & $0.90(\mathrm{P}<0.001)$ & & $0.89(\mathrm{P}<0.001)$ \\
Method 3 & $0.10(\mathrm{P}=0.23)$ & $0.62(\mathrm{P}<0.001)$ & $0.70(\mathrm{P}<0.001)$ & \\
\hline
\end{tabular}

Values in parentheses give P values from permutation tests. Values below the diagonal are for species richness; above the diagonal are species composition. Rows and columns listed as 'count' contain the number of studies of each pair of taxa in the dataset. 


\section{DISCUSSION}

Methods for accurately estimating the distribution of biodiversity are critically needed for efficient ecosystem management (Lindenmayer et al. 2015). Further, understanding the mechanisms that underpin these associations would resolve major research gaps in the field of community assembly (McGill et al. 2015). Consequently, the search for robust and transferable biodiversity surrogates has received much research attention, but with limited success (Gaspar et al. 2010, Heino 2010, Hess et al. 2006, Rodrigues and Brooks 2007). In this paper, we have presented the first synthesis of all pairwise cross-taxon congruence estimates between a number of plant and animal taxa. Our approach generalizes earlier research, by placing phenomena that have previously been studied in isolation within a common conceptual and analytical framework. This includes research on the scale-dependence of biodiversity congruence relationships (Hess et al. 2006, Westgate et al. 2014), or the efficacy of individual taxa as biodiversity surrogates (Butchart et al. 2012, Castagneyrol and Jactel 2012, Eglington et al. 2012). Our key finding was that birds and vascular plants outperform a range of alternative taxa as surrogates for the richness and composition of unmeasured taxonomic groups. Although similar patterns have been hypothesized before (Barreto de Andrade et al. 2014, Barton et al. 2014, Butchart et al. 2012, Eglington et al. 2012), our study is the first synthetic work to compare these assertions against alternative potential surrogates. Below we discuss the remainder of our results and their implications for improved biodiversity assessment.

\section{Selecting optimal biodiversity surrogates}

We found that sets of taxa that displayed high cross-taxon congruence also had complementary traits. Averaged across species richness and composition, for example, the taxa with the largest number of strong connections were Tracheophyta, Lepidoptera, Exopterygota and Aves (Fig. 2). These taxa are all widespread, easily detected, and taxonomically and functionally diverse (each of which can influence cross-taxon congruence; Larsen et al. 2009). Further, each of these taxa utilizes resources at a spatial scale that differs from the remaining groups, which may influence their collective utility as complementary biodiversity surrogates by capturing variability in habitat suitability at both micro- and macro- scales. However, understanding the precise mechanisms that underpin such patterns of cross-taxon congruence is difficult through meta-analysis, and even more so at high levels of he taxonomic hierarchy. It is also important that taxa with large numbers of strong associations were not always selected as optimal surrogates (e.g. Lepidoptera, Exopterygota; see Fig. 2). This was because the information they provided on unmeasured taxa had already been provided by previously selected groups (i.e. Aves and Plantae; see also Tulloch et al. 2013). Therefore, further work is warranted into the kinds of species traits and ecological interactions that promote or constrain crosstaxon congruence (Dehling et al. 2014, Velghe and Gregory-Eaves 2013), the conditions that influence these relationships (Chamberlain et al. 2014), and their implications for identifying optimal surrogates (Tulloch et al. 2016). 
We found strong study-dependent effects of spatial extent, grain size and location on congruence, but these effects had a strong influence on the choice of optimal taxon only when investigating species richness. Moreover, the results of our analysis were more congruent between metrics (richness vs. composition) once study-related variation was accounted for (Fig. 3h). Put differently, the patterns of cross-taxon congruence in species richness that we have described (Fig. 2a-c) could easily by 'swamped' by location- and study- specific variation. This expands on research showing that scale can profoundly influence observed patterns of richness (Dumbrell et al. 2008) and that species richness should be avoided as a metric for informing spatial prioritization (Brown et al. 2015). In our case, even significantly correlated estimates of pairwise congruence (i.e. from methods 1-3; see Table 2) resulted in different optimal surrogates for the richness of other taxa (Fig. 3). In contrast, congruence in species composition was relatively robust to study-related effects. That is, study-related parameters influenced the strength and variance of cross-taxon congruence relationships (Table 1), but had little influence on the ranking of optimal taxa (Fig. 2). Therefore, investigating richness and composition simultaneously, and testing the potential influence of scale on both metrics, are useful methods to help practitioners identify robust biodiversity surrogates.

Our results identify several new insights - as well as some potential pitfalls - for practitioners whose goal is to select optimal biodiversity surrogates. First, not all 'highly congruent' taxa were selected as complementary surrogates, because they often explained overlapping variation in the remaining (unmeasured) taxonomic groups (Tulloch et al. 2013). Instead, as the number of taxa that could be selected increased (i.e. at higher budgets), the algorithm often selected some taxa with weak pairwise associations, because they provided 'unique' information that could not be represented by other taxa. For example, using congruence estimates that account for differences in the spatial attributes and study design of each study (method 3), we would initially select Plantae and Aves as surrogates for the composition of all other taxa (Fig. 3i), but our third choice would be Mammalia which are poorly associated with other taxa in this dataset (Fig. 2f). Therefore, previous emphasis on identifying strong associations between taxa in the surrogate ecology literature is insufficient, as weak associations between taxa can be important in some instances. A second key issue is that the optimal choice of biodiversity surrogate is dependent on the target, with different taxa being selected depending on whether one wished to optimally detect invertebrates, vertebrates, or all taxa (Fig. 2). This is critical as monitoring and management programs often fail to set clear and measurable objectives (Lindenmayer and Likens 2009).

Finally, we suggest that practitioners should be explicit about the extent to which they expect prior information on cross-taxon congruence (either from this article, from research and monitoring in their own study area, or work from other locations) to help them identify candidate biodiversity surrogates in their study location (see also Canessa et al. 2015). These predictions can then be systematically improved over time 
using Bayesian principles, as is common in adaptive management (Westgate et al. 2013) and adaptive monitoring (Lindenmayer and Likens 2009). For example, systematic methods for updating users' relative confidence in competing models of system behavior over time have been widely used in the management of harvested populations for many years (e.g. Johnson et al. 2015, McGowan et al. 2015). These recommendations would be particularly effective if incorporated into a surrogacy framework designed to handle trade-offs in environmental decision-making (e.g. Lindenmayer et al. 2015).

\section{Future research}

Our work suggests that several important research gaps remain in the literature on biodiversity surrogates. In particular, consistent methods for assessing complementarity between distinct taxonomic groups are urgently needed (Grantham et al. 2010, Rodrigues and Brooks 2007). Further, our research investigates crosstaxon congruence in richness and composition over space; but further research would be needed to determine whether the surrogates we have identified show consistent covariation over time (Tulloch et al. 2016), which is critical for the design of effective monitoring programs (Lindenmayer et al. 2015). Finally, we have almost certainly missed several taxa of potential interest, simply because our results are limited to those taxa that were relatively well represented in the literature. For example, several potentially important surrogate taxa are missing from our study (e.g. Fungi) because they are relatively poorly studied, and therefore not all pairs of associations with other taxa were available. We also amalgamated several orders or classes to higher taxonomic levels to allow direct comparison in our study (Fig. 1b); such as merging Tracheophyta, Marchantiophyta and Bryophyta into the group 'Plantae', and several orders (including the Blattodea, Hemiptera \& Orthoptera) into the Superorder 'Exopterygota'. Rarely-studied taxa often have traits that ensure they are not assisted by conservation efforts aimed at the needs of commonly studied taxa (Darwall et al. 2011), meaning that data scarcity has important implications for biodiversity conservation. A small number of studies of these rarely-investigated taxa could have considerable potential for identifying important congruence relationships. We also assumed identical survey costs between taxa in this study, and so work is particularly needed to identify circumstances where the considerable costs of further research on cryptic taxa would improve our understanding of biodiversity sufficiently to justify their expense (e.g. Gardner et al. 2008, Kessler et al. 2011).

Our pairwise approach also highlights the potential utility of network theory (Boccaletti et al. 2006) for understanding patterns of spatial association within ecosystems. Network approaches are becoming increasingly prevalent in ecology, and while more commonly used to discuss functional associations between taxa such as trophic or pollination interactions (Ings et al. 2009), they also have been used to investigate spatial associations between taxa (e.g. Barberan et al. 2012, Tulloch et al. 2016, Wisz et al. 2013). In this case, we observed very few strong associations between taxa but many weak ones (Fig. 2a, c), a pattern that has been observed 
previously in other biological systems (e.g. in neurological networks: see Schneidman et al. 2006, Song et al. 2005) as well as elsewhere in ecology (McGill 2010). This high degree of similarity between distinct systems suggests that further study is warranted on the utility of network approaches for understanding spatial associations between taxonomic units, and how this information can be used to make predictions about the distribution and trajectory of biodiversity.

\section{Conclusions}

We have examined patterns of cross-taxon congruence using results from a metaanalysis of the ecology and conservation literature. These results can be used to guide predictions about which taxa may represent valid biodiversity surrogates, or to identify potentially significant ecological associations between taxa that apply across a range of spatial scales. Future research should focus on addressing bias in the taxa (or sets of taxa) that are investigated for use as potential surrogates.

\section{ACKNOWLEDGEMENTS}

We thank the authors whose works provided data to the meta-analysis on which our conclusions are based. In particular, Y. Mandelik, V. Landeiro, J. Diffendorfer, J. Kluge, M. Kessler \& S. Dullinger provided extensive supplementary information not included in their original articles. This project was supported by an Australian Research Council Laureate Fellowship to DBL.

\section{REFERENCES}

Araujo, M. B. et al. 2004. Representing species in reserves from patterns of assemblage diversity. - J. Biogeogr. 31: 1037-1050.

Arnqvist, G. and Wooster, D. 1995. Meta-analysis: synthesizing research findings in ecology and evolution. - Trends Ecol. Evol. 10: 236-240.

Balmford, A. and Gaston, K. J. 1999. Why biodiversity surveys are good value. - Nature 398: 204205.

Barberan, A. et al. 2012. Using network analysis to explore co-occurrence patterns in soil microbial communities. - ISME Journal 6: 343-351.

Barlow, J. et al. 2007. Quantifying the biodiversity value of tropical primary, secondary, and plantation forests. — Proc. Natl. Acad. Sci. U.S.A. 104: 18555-18560.

Barreto de Andrade, R. et al. 2014. Biotic congruence in humid tropical forests: A multi-taxa examination of spatial distribution and responses to forest disturbance. - Ecol. Indic. 36: $572-581$.

Barton, P. S. et al. 2013. The spatial scaling of beta diversity. — Global Ecol. Biogeogr. 22: 639-647.

Barton, P. S. et al. 2014. Robustness of habitat-based surrogates of animal diversity: a multi-taxa comparison over time and after fire. - J. Appl. Ecol. 51: 1434-1443.

Boccaletti, S. et al. 2006. Complex networks: Structure and dynamics. — Phys. Rep. 424: 175-308. 
Brown, C. J. et al. 2015. Effective conservation requires clear objectives and prioritizing actions, not places or species. - Proc. Natl. Acad. Sci. U.S.A.

Butchart, S. H. M. et al. 2012. Protecting Important Sites for Biodiversity Contributes to Meeting Global Conservation Targets. — PLoS ONE 7: e32529.

Canessa, S. et al. 2015. When do we need more data? A primer on calculating the value of information for applied ecologists. — Methods Ecol. Evol. 6: 1219-1228.

Castagneyrol, B. and Jactel, H. 2012. Unraveling plant-animal diversity relationships: a metaregression analysis. - Ecology 93: 2115-2124.

Chadès, I. et al. 2015. Benefits of integrating complementarity into priority threat management. Conserv. Biol. 29: 525-536.

Chamberlain, S. A. et al. 2014. How context dependent are species interactions? - Ecol. Lett. 17: 881890.

Darwall, W. R. T. et al. 2011. Implications of bias in conservation research and investment for freshwater species. - Conserv. Lett. 4: 474-482.

Dehling, D. M. et al. 2014. Functional relationships beyond species richness patterns: trait matching in plant-bird mutualisms across scales. - Global Ecol. Biogeogr. 23: 1085-1093.

Dumbrell, A. J. et al. 2008. Changes in species diversity following habitat disturbance are dependent on spatial scale: theoretical and empirical evidence. - J. Appl. Ecol. 45: 1531-1539.

Eglington, S. M. et al. 2012. A meta-analysis of spatial relationships in species richness across taxa: Birds as indicators of wider biodiversity in temperate regions. - J. Nat. Conserv. 20: 301309.

Ekroos, J. et al. 2013. Correlations in species richness between taxa depend on habitat, scale and landscape context. — Ecol. Indic. 34: 528-535.

Faith, D. P. 2003. Environmental diversity (ED) as surrogate information for species-level biodiversity. - Ecography 26: 374-379.

Field, S. A. et al. 2004. Minimizing the cost of environmental management decisions by optimizing statistical thresholds. — Ecol. Lett. 7: 669-675.

Gardner, T. A. et al. 2008. The cost-effectiveness of biodiversity surveys in tropical forests. - Ecol. Lett. 11: 139-150.

Gaspar, C. et al. 2010. Arthropods as surrogates of diversity at different spatial scales. — Biol. Conserv. 143: 1287-1294.

Gaston, K. J. and Williams, P. H. 1993. Mapping the World's Species-The Higher Taxon Approach. Biodivers. Lett. 1: 2-8.

Grantham, H. S. et al. 2010. Effectiveness of Biodiversity Surrogates for Conservation Planning: Different Measures of Effectiveness Generate a Kaleidoscope of Variation. - PLoS ONE 5:

Hawkins, B. A. and Porter, E. E. 2003. Does herbivore diversity depend on plant diversity? The case of California butterflies. - Am. Nat. 161: 40-49.

Heino, J. 2010. Are indicator groups and cross-taxon congruence useful for predicting biodiversity in aquatic ecosystems? - Ecol. Indic. 10: 112-117.

Heino, J. 2014. Taxonomic surrogacy, numerical resolution and responses of stream macroinvertebrate communities to ecological gradients: Are the inferences transferable among regions? - Ecol. Indic. 36: 186-194. 
Hess, G. R. et al. 2006. Effectiveness of biodiversity indicators varies with extent, grain, and region. Biol. Conserv. 132: 448-457.

Hunter Jr, M. et al. 2016. Two roles for ecological surrogacy: Indicator surrogates and management surrogates. - Ecol. Indic. 63: 121-125.

Ings, T. C. et al. 2009. Review: Ecological networks - beyond food webs. - J. Anim. Ecol. 78: 253 269.

Johnson, F. A. et al. 2015. Multilevel Learning in the Adaptive Management of Waterfowl Harvests: 20 Years and Counting. - Wildlife Society Bulletin 39: 9-19.

Kessler, M. et al. 2011. Cost-effectiveness of plant and animal biodiversity indicators in tropical forest and agroforest habitats. - J. Appl. Ecol. 48: 330-339.

Landeiro, V. L. et al. 2012. How far can we go in simplifying biomonitoring assessments? An integrated analysis of taxonomic surrogacy, taxonomic sufficiency and numerical resolution in a megadiverse region. — Ecol. Indic. 23: 366-373.

Langford, E. et al. 2001. Is the Property of Being Positively Correlated Transitive? - The American Statistician 55: 322-325.

Larsen, F. W. et al. 2009. Indicator taxa revisited: useful for conservation planning? — Divers. Distrib. 15: $70-79$.

Lawler, J. J. et al. 2015. The theory behind, and the challenges of, conserving nature's stage in a time of rapid change. - Conserv. Biol. 29: 618-629.

Legendre, P. et al. 2015. Should the Mantel test be used in spatial analysis? - Methods Ecol. Evol. 6: 1239-1247.

Lewandowski, A. S. et al. 2010. The effectiveness of surrogate taxa for the representation of biodiversity. - Conserv. Biol. 24: 1367-1377.

Lindenmayer, D. et al. 2015. A new framework for selecting environmental surrogates. - Sci. Total Environ. 538: 1029-1038.

Lindenmayer, D. B. and Likens, G. E. 2009. Adaptive monitoring: a new paradigm for long-term research and monitoring. — Trends Ecol. Evol. 24: 482-486.

Lung, T. et al. 2014. Biodiversity Funds and Conservation Needs in the EU Under Climate Change. Conserv. Lett. 7: 390-400.

Martin, L. J. et al. 2012. Mapping where ecologists work: biases in the global distribution of terrestrial ecological observations. — Front. Ecol. Environ. 10: 195-201.

McArthur, M. A. et al. 2010. On the use of abiotic surrogates to describe marine benthic biodiversity. — Estuarine, Coastal and Shelf Science 88: 21-32.

McGeogh, M. A. 1998. The selection, testing and application of terrestrial insects as bioindicators. Biol. Rev. 73: 181-201.

McGill, B. J. 2010. Towards a unification of unified theories of biodiversity. — Ecol. Lett. 13: 627642.

McGill, B. J. et al. 2015. Fifteen forms of biodiversity trend in the Anthropocene. - Trends Ecol. Evol. 30: 104-113.

McGowan, C. P. et al. 2015. Implementation of a framework for multi-species, multi-objective adaptive management in Delaware Bay. - Biol. Conserv. 191: 759-769.

Mellin, C. et al. 2011. Effectiveness of Biological Surrogates for Predicting Patterns of Marine Biodiversity: A Global Meta-Analysis. — PLOS ONE 6: e20141. 
Moilanen, A. 2008. Generalized Complementarity and Mapping of the Concepts of Systematic Conservation Planning. - Conserv. Biol. 22: 1655-1658.

R Core Development Team 2015. R: A Language and Environment for Statistical Computing, Version 3.2.3. - R Foundation for Statistical Computing.

Rigby, R. A. and Stasinopoulos, D. M. 2005. Generalized additive models for location, scale and shape. - J. Roy. Statist. Soc. Ser. C 54: 507-554.

Rodrigues, A. S. L. and Brooks, T. M. 2007. Shortcuts for biodiversity conservation planning: The effectiveness of surrogates. - Annu. Rev. Ecol. Evol. Syst. 38: 713-737.

Sachs, J. D. et al. 2009. Biodiversity Conservation and the Millennium Development Goals. — Science 325: 1502-1503.

Sarkar, S. and Margules, C. 2002. Operationalizing biodiversity for conservation planning. — Journal of Biosciences 27: 299-308.

Schall, J. J. and Pianka, E. R. 1978. Geographical trends in numbers of species. — Science 201: 679686.

Schneidman, E. et al. 2006. Weak pairwise correlations imply strongly correlated network states in a neural population. - Nature 440: 1007-1012.

Song, S. et al. 2005. Highly Nonrandom Features of Synaptic Connectivity in Local Cortical Circuits. - PLoS Biol 3: e68.

Supp, S. R. and Ernest, S. K. M. 2014. Species-level and community-level responses to disturbance: a cross-community analysis. — Ecology 95: 1717-1723.

Tulloch, A. I. T. et al. 2016. Dynamic species co-occurrence networks require dynamic biodiversity surrogates. - Ecography

Tulloch, A. I. T. et al. 2013. Accounting for Complementarity to Maximize Monitoring Power for Species Management. — Conserv. Biol. 27: 988-999.

Velghe, K. and Gregory-Eaves, I. 2013. Body size is a significant predictor of congruency in species richness patterns: A meta-analysis of aquatic studies. — PLoS ONE 8: e57019.

Westgate, M. J. et al. 2014. Global meta-analysis reveals low consistency of biodiversity congruence relationships. — Nat. Commun. 5: 3899.

Westgate, M. J. et al. 2013. Adaptive management of biological systems: a review. — Biol. Conserv. 158: 128-139.

Wisz, M. S. et al. 2013. The role of biotic interactions in shaping distributions and realised assemblages of species: implications for species distribution modelling. — Biol. Rev. 88: 15-30.

Wolters, V. et al. 2006. Relationship among the species richness of different taxa. - Ecology 87: 1886-1895. 